\title{
Lifetime Enhancement of Cluster Based Wireless Sensor Network through Energy Efficient MAC Protocol
}

\author{
B. Priya ${ }^{1}{ }^{*}$, S. Solai Manohar ${ }^{2}$ \\ ${ }^{1}$ Department of Electronics and Communication Engineering, Rajalakshmi Engineering College, Chennai, India \\ ${ }^{2}$ Department of Electrical and Electronics Engineering, Anand Institute of Higher Technology, Chennai, India \\ Email: *shanth1@hotmail.com
}

Received 27 March 2016; accepted 20 April 2016; published 19 July 2016

Copyright (C) 2016 by authors and Scientific Research Publishing Inc.

This work is licensed under the Creative Commons Attribution International License (CC BY). http://creativecommons.org/licenses/by/4.0/

cC) (i) Open Access

\begin{abstract}
The efficient use of energy is an important performance metric to extend the lifetime of wireless sensor networks. Since major energy consumption of node is due to its transceiver, the design of MAC protocol plays a vital role in sensor network design. In cluster based sensor networks, due to the different functions of member node and cluster head node, the usage of common MAC protocol results increased energy consumption. To overcome this problem, a novel energy efficient hybrid MAC protocol (EEHMAC) for cluster based wireless sensor networks is proposed in this paper. The proposed EEHMAC protocol uses E-TDMA (Energy efficient TDMA) for intra-cluster communication and FDMA (Frequency Division Multiple Access) for inter-cluster communication. IDS (Iterative Deepening Search) based Scheduling algorithm is used for assigning time slot and frequency slot to nodes. Nodes in EEHMAC follow the periodic duty cycle, which reduces the idle listening, and overhearing problems. Simulation results reveal that an average of $18 \%$ energy saving is achieved compared to LEACH (Low Energy Adaptive Clustered Hierarchy) protocol and 10\% energy is saved in comparison with GH-MAC (Graph theory based Hybrid MAC) protocol. It is evident that delay of EEHMAC is reduced by $17 \%$ and throughput is increased by $15 \%$ under all traffic conditions. These results demonstrate that EEHMAC performs better than existing MAC protocols in terms of energy efficiency, delay and throughput.
\end{abstract}

\section{Keywords}

Channel Allocation, Delay Systems, Energy, Lifetime Estimation, Throughput, Wireless Sensor Network (WSN)

\footnotetext{
${ }^{*}$ Corresponding author.
} 


\section{Introduction}

A wireless sensor network (WSN) is a collection of a large number of low cost distributed sensor nodes to monitor physical or environmental conditions, such as temperature, sound, pressure, etc. and cooperatively pass their data through the network to a main location i.e., base station. These nodes are usually equipped with limited energy source. In majority of application scenarios, recharging or replacement of batteries are not possible because of its deployment in harsh and remote environment. This makes energy efficiency an important issue to extend the lifetime of sensor nodes. Because of these reasons, nowadays researchers are focusing on the development of energy aware protocols for wireless sensor networks [1]. Major energy consumption is due to the nodes' radio during their active period [2]. However, node's radio can be controlled with efficient design of channel access scheme. Hence, the design of MAC protocol plays a vital role in WSN design.

There are four major sources of energy waste in MAC protocol: collision, idle listening, overhearing and protocol overhead [3]. Various MAC protocols were designed to save lifetime of sensor node by solving these problems. In 802.11 MAC protocol, idle listening consumes 50\% to $100 \%$ of energy required for receiving [4]. Therefore, idle listening becomes most important factor of energy waste. Periodic wake-up scheduling where the nodes are in sleep mode or low power mode most of the time is the powerful concept used to overcome the idle listening problem. To take advantages of schedule based and contention based protocols, the concept of hybrid MAC protocols was introduced. To save the energy consumption, clustering concept is used in sensor networks. Normally, a single channel access scheme is used for entire sensor network. However, due to the various functions done by cluster head $(\mathrm{CH})$ node and member node the common MAC protocol for both, results in increased energy consumption. The analysis of different MAC protocols was studied in Section 2.

The rest of the paper is structured as follows: Section 3 emphasizes on the design of proposed EEHMAC protocol and the heuristic method to select the number of clusters. The results are presented in Section 4 following with the conclusion in Section 5.

\section{Related Works}

Energy management is an important research area in the sensor networks. S-MAC [4] and T-MAC [5] are the low duty cycle protocols proposed for maximum energy efficiency. In these protocols, energy efficiency is achieved by reducing idle listening. S-MAC uses periodical sleep to reduce energy consumption due to idle listening and uses message passing approach to delay. In T-MAC, the energy efficiency of S-MAC is improved by $50 \%-80 \%$ with adaptive wakeup scheduling. However, these protocols have the problem of asymmetric communication patterns. In [6], different wakeup scheduling is analyzed and method to increase energy efficiency is proposed. Here, multi-parent scheme that assigns multiple forwarding nodes to each node with different wakeup schedule is used. It increased the lifetime of sensor nodes from 40 months to 65 months without degrading the guaranteed delay performance. However, interference free scheduling is not provided in this method. ML-MAC [7] is a distributed contention-based MAC protocol designed for wireless sensor networks that uses periodic listen and sleep concept. ML-MAC divides the active period into L non-overlapping layers and nodes in each layer follows periodic listen/sleep schedule. Due to the short listening time of sensor nodes in ML-MAC, the energy consumption of data transmission is reduced to greater extend. Here, the idle listening time is reduced by the factor of number of layers used. However, delay is increased in this protocol due to the increased waiting time.

One palpable approach is to use either TDMA or FDMA for interference free scheduling. Nevertheless, TDMA has many other disadvantages [8] like clock synchronization, handling of dynamic topological changes, collision free scheduling etc. The multi channel MAC protocols have disadvantages like broadcast support, network partitioning, channel switching etc. as listed in [9]. HyMAC [10] is a hybrid TDMA/FDMA protocol suitable for WSN applications, designed to provide high throughput and small bounded end-to-end delay. It achieved higher throughput by using simultaneous data transmission with multiple frequencies. However, this protocol always works in the hybrid mode leads to increased energy consumption under low load conditions. Z-MAC [11] is another hybrid MAC protocol, which combines the strength of CSMA and TDMA for better energy efficiency and improved channel utilization. The main feature of Z-MAC is its adaptability to different contention levels of network. However, this protocol is suffered by synchronization problem and not suitable for cluster based sensor networks.

In energy efficient hybrid MAC protocol for cluster based network [12], the BMA (Bit Map Assisted) protocol is used for intra cluster communication and nanoMAC protocol is used for inter cluster communication. 25\% 
energy reduction is achieved by using BMA and 40\% energy consumption is reduced with nanoMAC. GH-MAC [13] is another hybrid MAC protocol designed for cluster based sensor networks to increase the lifetime and to decrease delay. In this protocol, G-ETDMA (Game theory based energy efficient TDMA) and G-nanoMAC (Game theory based nanoMAC) are used for intra-cluster communication and inter-cluster communication respectively. By using repeated game concept, the energy consumption is estimated in GH-MAC for various load conditions. Compared to E-TDMA, 22\% energy consumption is reduced in G-ETDMA due to the avoidance of idle listening. Compared to nanoMAC, 1.6 times less energy is consumed in G-nanoMAC. However, the delay performance is not improved in theses protocols for various traffic loads.

To solve this issue, an energy efficient hybrid MAC protocol (EEHMAC) that considers the characteristics of sensor node is proposed in this paper. The proposed EEHMAC uses TDMA for intra-cluster communication since the member nodes required to transmit minimum data for short duration. Since $\mathrm{CH}$ nodes are required to transmit maximum data for long duration, FDMA is used for inter-cluster communication. To avoid inter channel interference, the number of frequencies is limited to number of clusters and frequency reuse concept is utilized in the proposed protocol. A heuristic method is used to select the optimum number of clusters for varying network density. With the help of simultaneous data transmission, delay of sensor node is reduced to greater extend and energy efficiency is improved. The performance of proposed EEHMAC protocol is estimated in terms of energy efficiency, delay and throughput.

\section{EEHMAC Protocol}

The main goal of EEHMAC protocol is to reduce energy consumption and delay, while increasing throughput. By combining the advantages of TDMA and FDMA, the proposed protocol achieves better energy efficiency compared to LEACH and GH-MAC. This protocol is suitable for applications in which data gathered by sensor nodes are delivered to destination in a timely manner.

\subsection{System Model}

In a cluster based wireless sensor network two types of communication involves namely inter-cluster and intracluster communication. Nodes sense the environment and transmit data to the Cluster Head (CH) during intracluster communication and $\mathrm{CH}$ communicates to the base station and other $\mathrm{CH}$ during inter-cluster communication. In intra-cluster communication, the amount of data transmitted is limited but the contention is more. Therefore, the active period is divided into "S" number of slots and is assigned to all users using TDMA approach. In inter-cluster communication, cluster head transmits more data, which is collected from its member nodes. Therefore, the entire active period is given to the $\mathrm{CH}$ nodes with "N" different frequencies using FDMA concept. The system model of proposed protocol is shown in Figure 1. The duty cycle member node in EEHMAC for intra-cluster communication is shown in Figure 2(a). The active period comprises of multiple time slots that are assigned to member nodes. Figure 2(b) shows the duty cycle of $\mathrm{CH}$ node during inter-cluster communication. Here, the active period is divided with respect to frequency and each $\mathrm{CH}$ is assigned with one frequency slot.

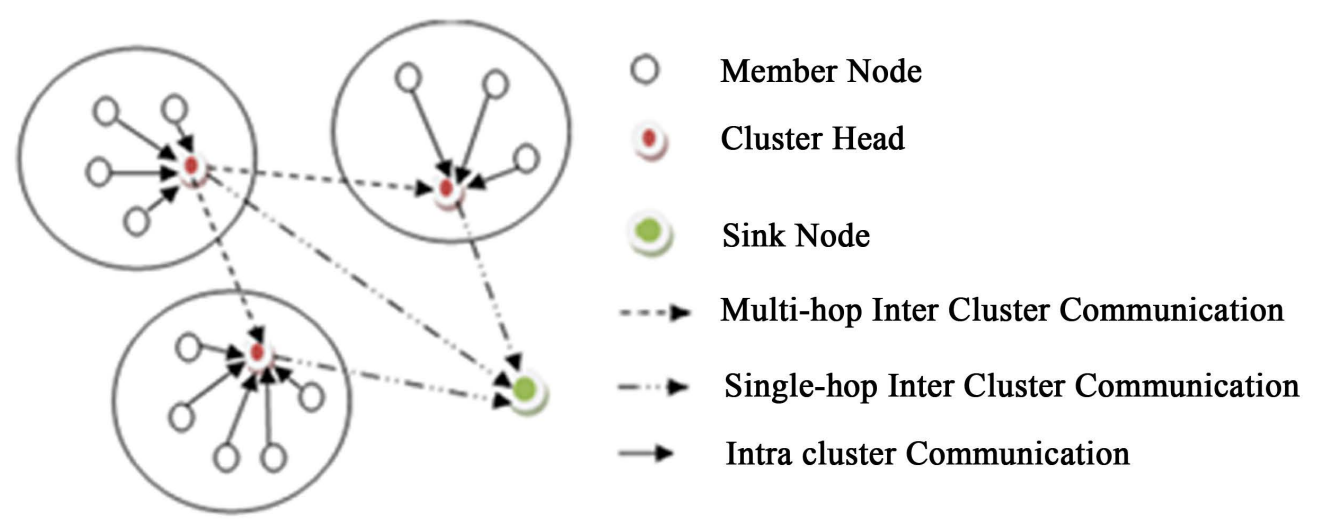

Figure 1. System model. 


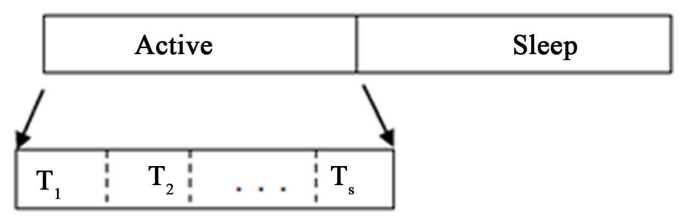

(a)

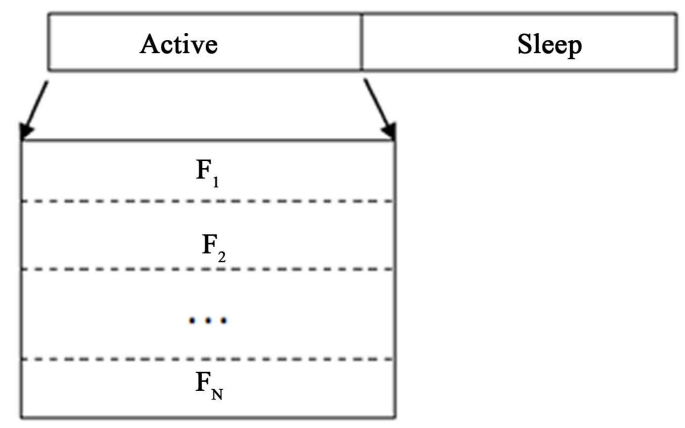

(b)

Figure 2. (a) Duty cycle of a $\mathrm{CH}$ Intra-cluster communication; (b) Duty cycle of $\mathrm{CH}$ in inter-cluster communication.

\subsection{Hybrid MAC Protocol}

The hybrid MAC protocol is organized into rounds and each round is subdivided into setup phase and steady state phase. During the setup phase, $\mathrm{CH}$ is selected based on the energy level and the selected $\mathrm{CH}$ will broadcast the advertisement message. The nodes that can reach the cluster head with minimum energy will join in that cluster. After forming the cluster, $\mathrm{CH}$ will assign the TDMA slot and forward it to the member nodes. Then the system enters into the steady state phase. During the assigned time slot, the node will be in the active state and goes to sleep state otherwise.

Based on the advertisement message broadcasted by the $\mathrm{CH}$, sink will assign the frequency slot to the $\mathrm{CH}$ and broadcast during the cluster setup phase. The frame structure of hybrid MAC is shown in Figure 3. The frame is divided into three fields. The node status field is used to carry the information about node status (i.e.) whether the node has sensed data for transmission or not. If the node does not have data, then the $\mathrm{CH}$ will assign the time slot to other nodes that have data. During intra-cluster communication filed, the time is divided into number of time slots and is assigned to member nodes. During the active state if the node does not have data to transmit/receive then immediately it goes to sleep state. Thus, the designed EEHMAC protocol reduces idle listening and overhearing problems of MAC protocol. In the inter-cluster communication field, the entire period is used by the $\mathrm{CH}$ for data transmission with different frequencies.

\subsection{Time Slot and Frequency Slot Assignment}

The network is using ISM band. The entire bandwidth $(2.4 \mathrm{GHz})$ is divided into two parts in the ratio of 1:3. Lower part of the bandwidth is $0.8 \mathrm{GHz}$ and is used for intra-cluster communication. Upper part of the bandwidth is $1.6 \mathrm{GHz}$ and is used for inter-cluster communication. The network is modeled as a graph $\mathrm{G}=(\mathrm{V}, \mathrm{E})$, in which $\mathrm{V}$ is the set of nodes and $\mathrm{E}$ represents the link between the nodes. If the nodes $\mathrm{i}$ and $\mathrm{j}$ are in the communication range then there exists $(i, j) \in E$ and $(j, i) \in E$. Based on the relationship between scheduling algorithm and graph theory, NP-hard coloring problem can be used to assign the time slots to the nodes [14]. Slot assigned to one node should not conflict with its one-hop and two-hop neighbor. Therefore, all nodes can transmit/receive data from its neighbors without conflict. A time slot $\mathrm{T}_{\mathrm{k}}$ can be assigned to node i if $\mathrm{T}_{\mathrm{k}} \notin \mathrm{T}\left(N_{i}^{1}\right) \cup \mathrm{T}$ $\left(N_{i}^{2}\right)$. Here T $\left(N_{i}^{1}\right)$ and $\mathrm{T}\left(N_{i}^{2}\right)$ represents the time slot assigned to node i's one-hop and two-hop neighbors respectively.

Here the IDS (Iterative Deepening Search) algorithm is used for slot assignment. It combines the space efficiency of Depth First Search (DFS) with the optimality of Breath First Search (BFS) algorithm. For node time slot assignment the scheduling algorithm performs the IDS constructing a tree having cluster head as its root. As each node, $i$ is traversed by IDS, it is assigned a default time slot. For frequency slot assignment the scheduling algorithm performs IDS constructing sink node as its root. Figure 4 shows the operation of scheduling algorithm. In this, $\mathrm{f} 1, \mathrm{f} 2$ and $\mathrm{f} 3$ shows assigned frequency slots of cluster head nodes while the $\mathrm{t} 1$, $\mathrm{t} 2$ and $\mathrm{t} 3$ shows the time slots assigned to member nodes. Note that such an assignment allows the nodes to transmit data packets without any collision. Frequency assigned for one $\mathrm{CH}$ is not assigned for another $\mathrm{CH}$. Therefore, it is mandatory to determine the number of frequencies required for inter-cluster communication. To avoid collision in frequency 


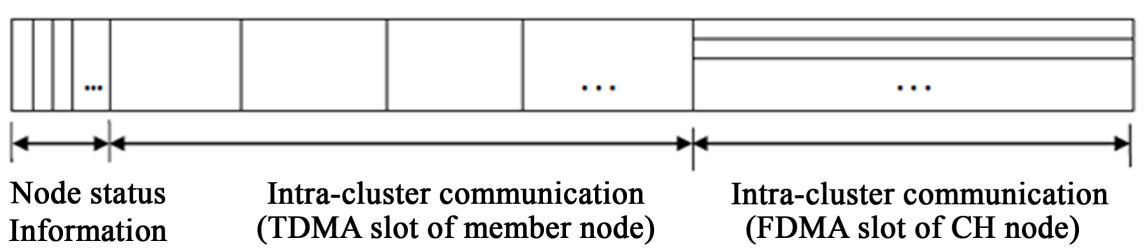

Figure 3. Frame structure of Hybrid MAC.

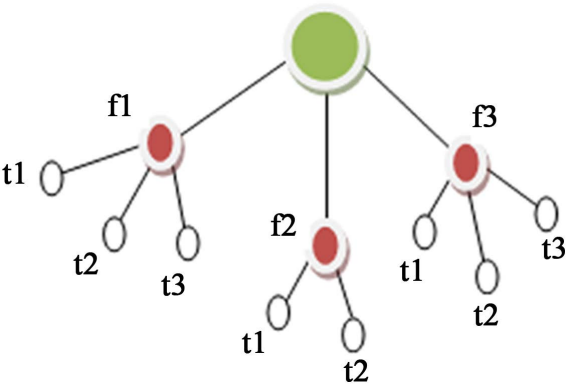

Figure 4. Operation of scheduling algorithm.

slots, one $\mathrm{CH}$ is assigned with one frequency. Therefore, the optimum number of clusters in the network will decide the number of frequencies required.

\subsection{Optimum Number of Frequencies}

A simple model, in which the transmitter dissipates energy to run radio electronics and the power amplifier and the receiver dissipates energy to run radio electronics is considered [15] for calculation of optimum number of frequencies required. Figure 5 describes the energy dissipation model of sensor node radio.

In the data collection process, first the node will send data to the cluster head and from there the data is forwarded to sink node. Therefore, the total energy spent for data transmission is calculated by combining the energy consumed by member node and by head node.

$$
E_{\text {totalframe }}=E_{C H}+E_{M N}
$$

Energy spent by the member node to transmit a packet of $m$ bits to its head node of distance $d$ is given by

$$
E_{t x}(m, d)=m E_{\text {elec }}+m E_{r a} d^{2}
$$

where $E_{\text {elec }}$ is the energy depleted per bit to operate the transceiver circuit, $E_{r a}$ is the radio amplifier energy. Apparently, the distance between the member node and its c luster head is less than the crossover distance, so the energy dissipation follows the Friss free space model (i.e.) $d^{2}$ power loss. The network consists of $N$ nodes distributed uniformly in an $M \times M$ region. Network is divided into k number of circle clusters and the distance from head node to member node is given by

$$
E\left(d^{2}\right)=\frac{M^{2}}{2 \pi k}
$$

The total energy spent by the member node is given by

$$
E_{M N}=E_{t x}(m, d)=m E_{\text {elec }}+m E_{r a} \frac{M^{2}}{2 \pi k}
$$

Each cluster head will dissipates energy by receiving data from member nodes, by data aggregation and by transmitting the aggregated data to sink node. Thus, the energy spent by the head node during single frame is given by,

$$
E_{C H}=\left(\frac{N}{k}-1\right) E_{r x}(m, d)+\left(\frac{N}{k}-1\right) E_{d a}+E_{t x}(m, d)
$$




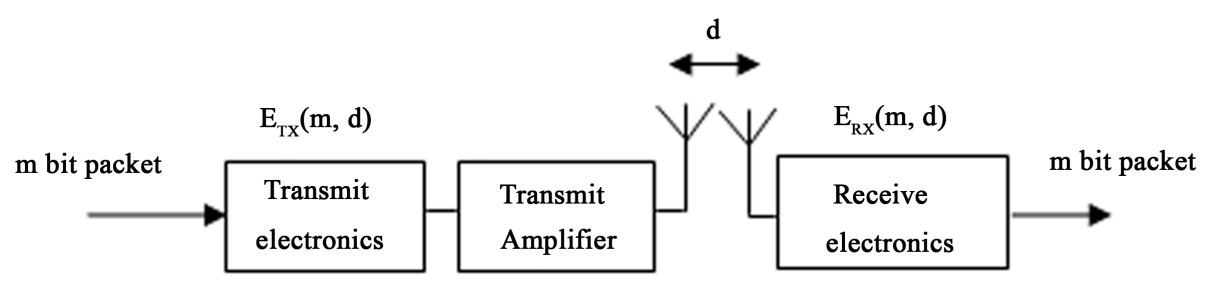

Figure 5. Radio energy dissipation model.

In this case, the distance from cluster head node to base station is greater than the cross over distance. Therefore, energy dissipation follows the two-ray ground model (i.e.) $d^{4}$ power loss.

$$
E_{C H}=\left(\frac{N}{k}-1\right) E_{r x}(m, d)+\left(\frac{N}{k}-1\right) E_{d a}+m E_{\text {elec }}+m E_{T R A} d^{4}
$$

where $E_{d a}$ is the data aggregation energy and $E_{T R A}$ is the transmitter amplifier energy. The total energy spent by the cluster is given by

$$
E_{c}=E_{C H}+\left(\frac{N}{k}-1\right) E_{M N}
$$

By substituting the Equation (6) and Equation (4) in Equation (7), we get

$$
E_{c}=\left(\frac{N}{k}-1\right) E_{r x}(m, d)+\left(\frac{N}{k}-1\right) E_{d a}+m E_{\text {elec }}+m E_{T R A} d^{4}+\left(\frac{N}{k}-1\right)\left[m E_{\text {elec }}+m E_{r a} \frac{M^{2}}{2 \pi k}\right]
$$

The total energy consumed by the network is given by

$$
E_{t}=k E_{c}=k\left\{\left(\frac{N}{k}-1\right) E_{r x}(m, d)+\left(\frac{N}{k}-1\right) E_{d a}+m E_{\text {elec }}+m E_{T R A} d^{4}+\left(\frac{N}{k}-1\right)\left[m E_{\text {elec }}+m E_{r a} \frac{M^{2}}{2 \pi k}\right]\right\}
$$

We can find the optimum number of frequencies by calculating optimum number of clusters. This can be done by setting the derivative of total energy with respect to $\mathrm{k}$ to zero.

$$
\begin{aligned}
& \frac{\mathrm{d} E_{t}}{\mathrm{~d} k}=0 \\
& k=\sqrt{\frac{N m E_{r a} M^{2}}{2 \pi\left(m E_{\text {TRA }} d^{4}-m E_{\text {elec }}-E_{d a}\right)}}
\end{aligned}
$$

By using the Equation (10), the number of frequencies (clusters) required is calculated. If the optimum number of clusters is increased then the energy consumed by the network will be reduced and thereby increasing lifetime of network.

\subsection{Energy and Delay Analysis}

Equation (9) defines the energy consumed by the network in a single frame and the total energy consumption is given by Equation (11).

$$
E_{\text {total }}=E_{t} N_{f}
$$

where $N_{f}$ represents the number of frames. In sensor networks, data collection is a time-sensitive function. Therefore, it is important to receive the data in a timely manner. Here, delay is defined as the time taken to transmit the data successfully. In intra-cluster communication, the member node has to wait for node status information and then only it can transmit the data. Therefore, the delay for intra-cluster communication is given by,

$$
D=\left(\frac{N}{k}-1\right) t_{s}+t_{d C H}
$$


During inter-cluster communication, the $\mathrm{CH}$ will transmit the data after collecting it from the member nodes. So, the delay for inter-cluster communication is given by,

$$
D=\left(\frac{N}{k}-1\right) t_{r}+\left(\frac{N}{k}-1\right) t_{a g}+t_{d B S}
$$

where $t_{s}, t_{r}$ and $t_{a g}$ represents time taken for node status information, data reception and data aggregation respectively. Distance from member node to $\mathrm{CH}$ node is smaller than the distance between $\mathrm{CH}$ node and base station. Therefore, different timings are used like $t_{d C H}$ and $t_{d B S}$.

\section{Performance Evaluation}

This section evaluates the energy consumption, end-to-end delay and throughput for various schemes using MATLAB simulation tool. Since LEACH is the basic protocol developed for cluster based networks, the performance of EEHMAC is compared with it. GH-MAC is cluster based MAC protocol that uses adaptive MAC scheme for inter and intra-cluster communication. Therefore, the results of EEHMAC are compared with GH-MAC also. The parameters used for simulation are given in Table 1. Simulation is carried out for $1000 \mathrm{~s}$. Random distribution of 100-node network is shown in Figure 6.

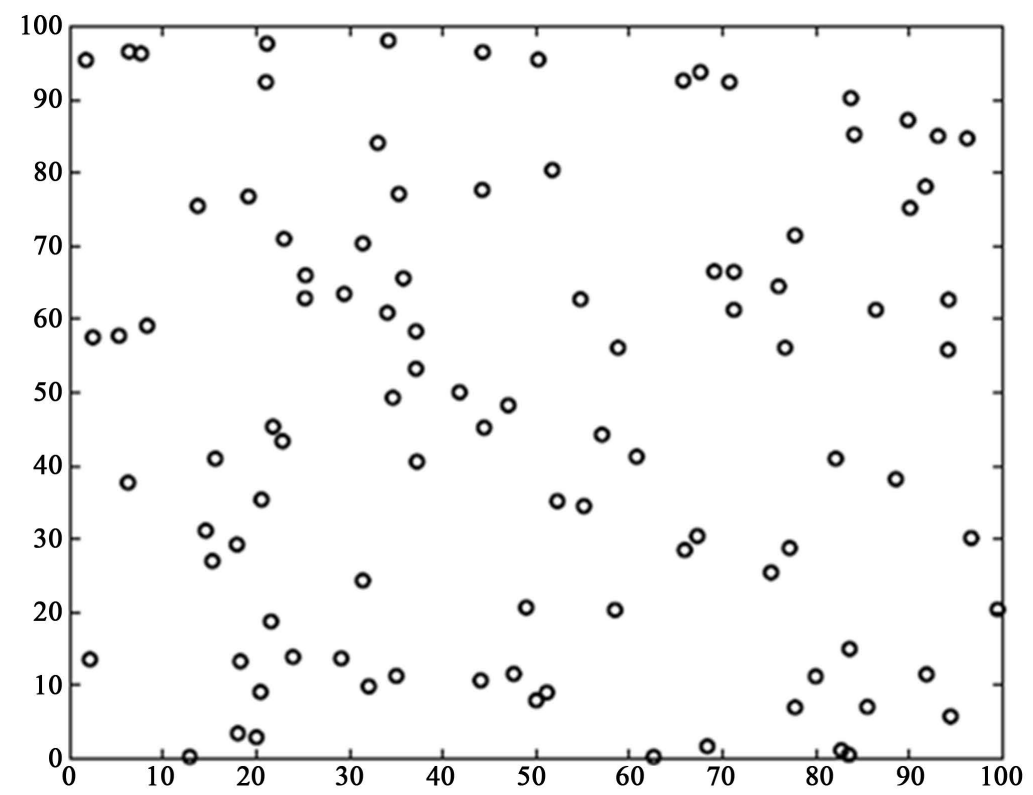

Figure 6. Random distribution of 100-node Network.

Table 1. Simulation parameters.

\begin{tabular}{cc}
\hline Parameters & Values \\
No. of nodes (N) & $100-1000$ \\
Topology & Uniform, Random \\
Size & $100 \times 100$ \\
Distance to base station & $100 \mathrm{~m} \mathrm{-} 250 \mathrm{~m}$ \\
Number of frequencies & $1-14$ \\
Eda & $5 \mathrm{~nJ} / \mathrm{bit}$ \\
Packet size (m) & $500 \mathrm{bytes}$ \\
Eelec & $50 \mathrm{~nJ} / \mathrm{bit}$ \\
Efs & $10 \mathrm{pJ} / \mathrm{bit}$ \\
E $_{\mathrm{TRA}}$ & $0.0013 \mathrm{pJ} / \mathrm{bit}$ \\
\hline
\end{tabular}




\subsection{Optimum Number of Clusters}

Since the number of frequencies used in the proposed system depends on the number of clusters in the network, the optimum number of clusters required is calculated and compared with conventional cluster based protocol LEACH. Simulation is run by varying network density from 100 to 1000 and the results are shown in Figure 7. From Figure 7, it is evident that, proposed protocol uses less number of clusters compared to existing protocol. If number of clusters is increased then the inter-cluster energy required to transmit data to the base station will be increased. Thus, in proposed EEHMAC protocol, the inter-cluster energy is reduced by reducing number of clusters. For 1000 nodes, EEHMAC uses 7 clusters whereas LEACH uses 11 clusters. Thus by using proposed protocol, we can save network lifetime about 10\% - 15\%. By varying the distance between node and base station, the optimum number of clusters required for the EEHMAC protocol is calculated and is shown in Figure 8. If the distance is increased then the number of clusters required for effective communication is reduced. For a distance of $100 \mathrm{~m}$ the maximum number of clusters used in the proposed system is 5 when the network density is 500 .

An average energy spent by the nodes for different number of clusters is calculated for EEHMAC and Figure 9 shows the results in comparison with LEACH protocol. Energy consumption is reduced for increase in number

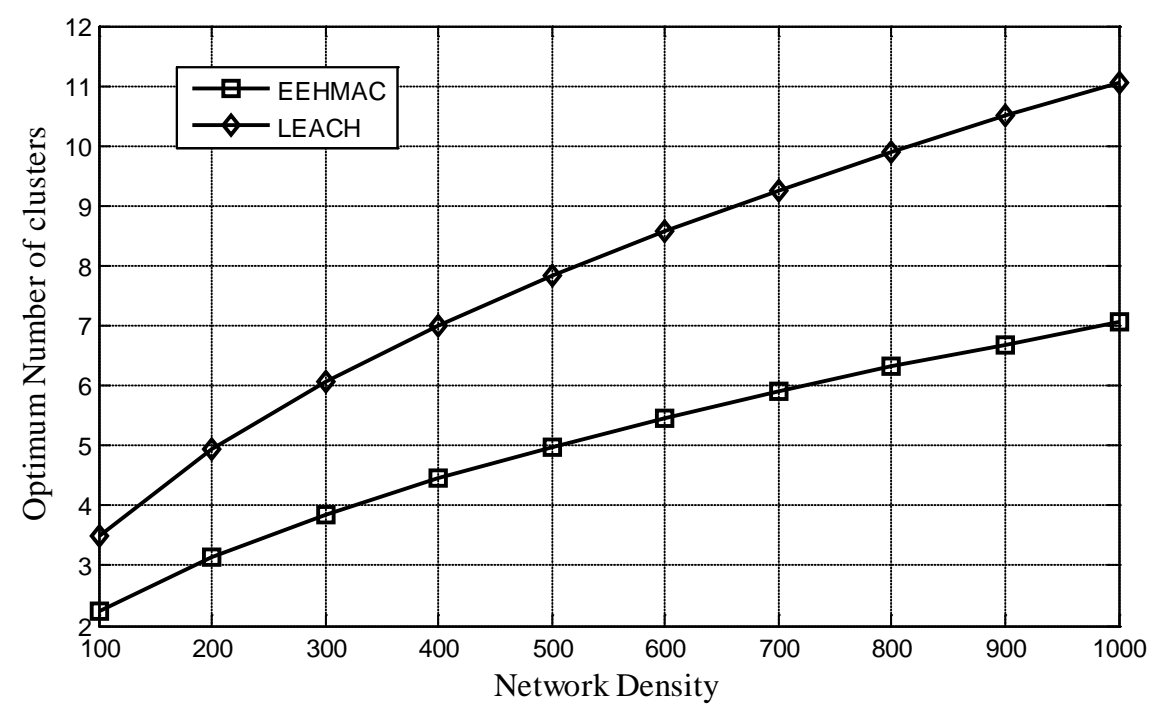

Figure 7. Optimum number of clusters for various network densities.

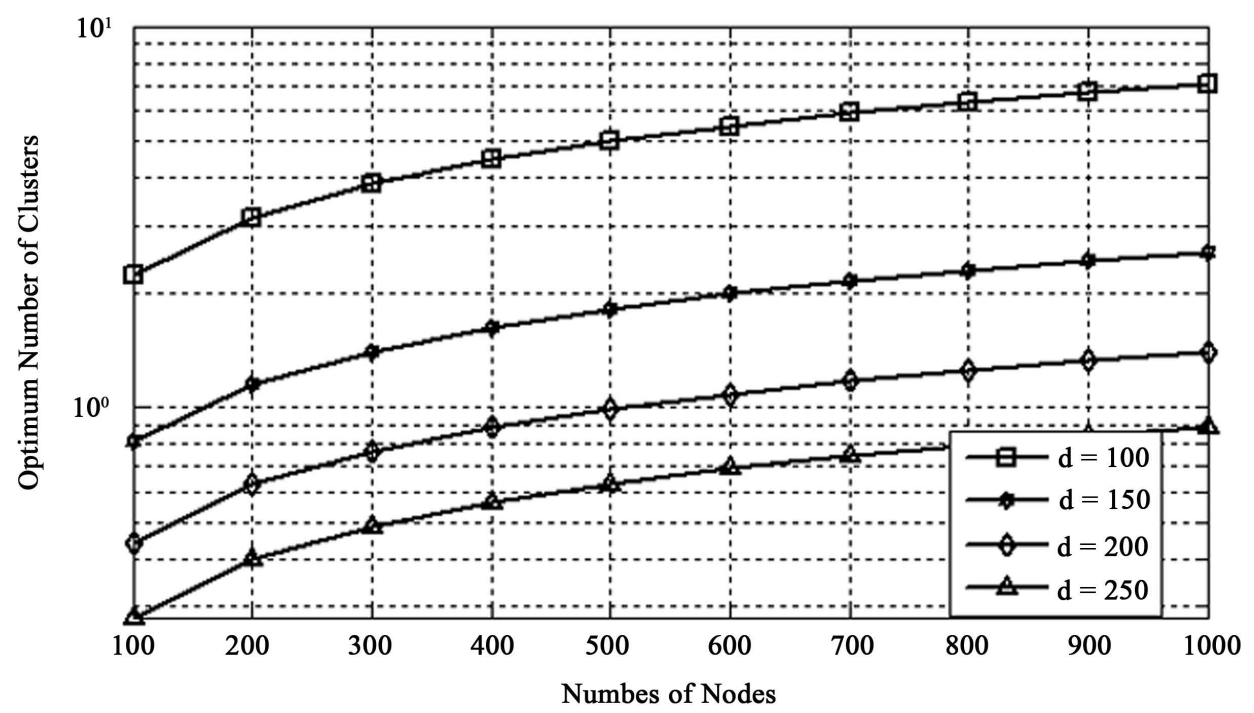

Figure 8. Optimum number of clusters for various base station distance. 


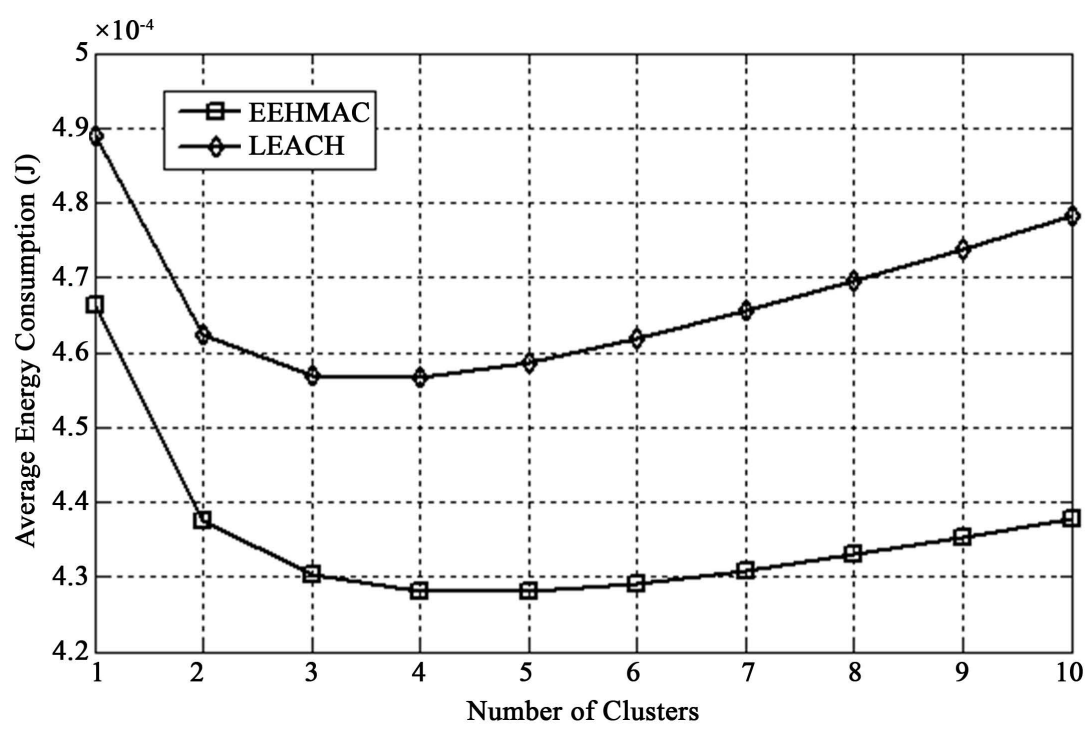

Figure 9. Average energy consumption for various number of clusters.

of clusters and increased if the number of clusters exceeded the optimum value. If numbers of clusters are less than the optimum size then more number of member nodes has to transmit data for long distance to reach $\mathrm{CH}$ node. This results in depletion of node's energy. When the numbers of clusters are more than optimum value, then the number of transmissions done by the $\mathrm{CH}$ to the base station is greater than data aggregation process and leads to increased energy consumption. The result shown in Figure 9 demonstrates that optimum number of clusters should be 4 to 5 for energy efficient operation in 100-node network. In this case, EEHMAC saves energy up to $7 \%$ than LEACH. In worst case, when the number of clusters are more than optimum size EEHMAC reduces the energy consumption by $10 \%$. These results prove that lifetime of sensor network is increased by an average of $7 \%$ in EEHMAC compared to LEACH.

For the network having 1000 nodes, total energy consumed by the EEHMAC protocol is calculated by varying the number of frequency slots, and the result is shown in Figure 10. This graph shows that the total energy consumption is reduced in EEHMAC if more number of frequencies is used. Since, multiple $\mathrm{CH}$ transmit data simultaneously using different frequencies, the possibility of collision is reduced. This results in reduced energy consumption. However, in the proposed protocol to reduce the inter channel interference the numbers of frequency slots used are limited to optimum value of number of clusters. For 1000 node network, the optimum number of clusters is 14 and is equal to number of frequency slots.

\subsection{Energy Analysis}

The total energy consumed by the nodes in LEACH, GH-MAC and EEHMAC protocols for various network densities is simulated and compared in Figure 11. This graph shows that EEHMAC provide better performance in terms of energy than existing protocols. The reason is that EEHMAC uses the hybrid of TDMA and FDMA protocol which greatly enables the simultaneous transmission of different clusters and also during inter-cluster communication, and intra-cluster communication is also possible. When the network density is 100 , the EEHMAC saves energy by an amount of 50\% and 47\% compared to LEACH and GHMAC respectively. For 1000-node network, the energy consumption is reduced in EEHMAC by 58\% when compared to LEACH protocol and $45 \%$ in comparison with GHMAC protocol. Thus, by an average EEHMAC saves the energy consumption of sensor nodes by 56.3\% and $45.23 \%$ in comparison with LEACH and GHMAC protocols. Thus, under all load conditions EEHMAC proved to be energy efficient compared to conventional MAC protocols LEACH and GHMAC.

Figure 12 shows the frame energy for LEACH, GHMAC and EEHMAC protocols. It is observed that frame energy consumption for proposed protocol is lesser than that of existing protocols. In GHMAC for inter-cluster communication each time the $\mathrm{CH}$ node has to do CSMA, leads to additional overhead and increased energy consumption. Whereas the usage of fixed frequency slots for inter-cluster communication in EEHMAC reduced 


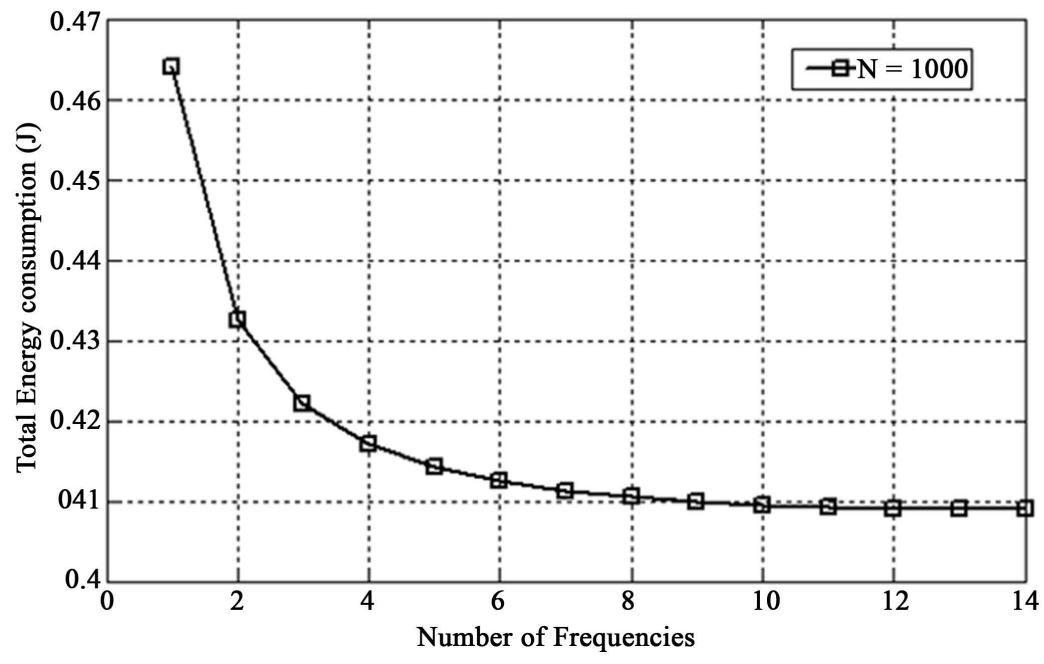

Figure 10. Energy consumption for different frequency slots.

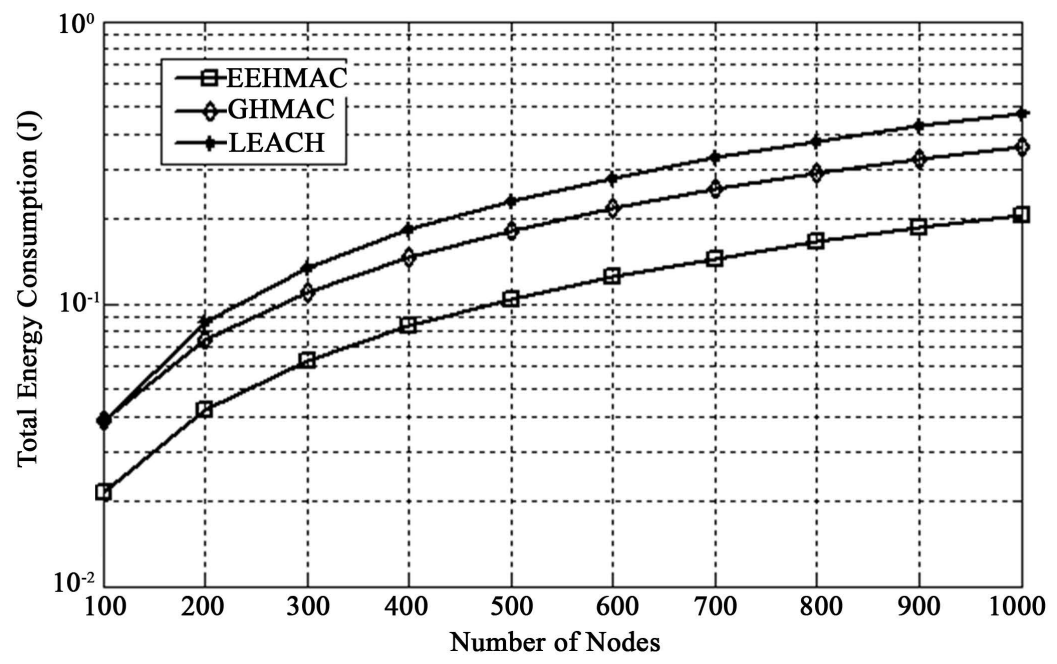

Figure 11. Energy consumption for various network densities.

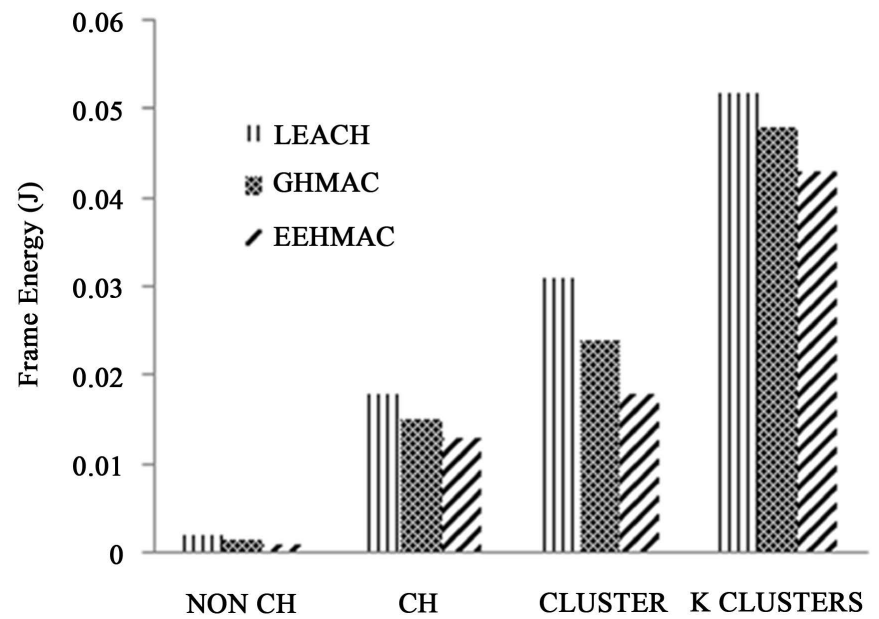

Figure 12. Frame energy for various protocols. 
the protocol overhead and results in reduced energy consumption. The proposed EEHMAC protocol reduced the frame energy for k clusters by $23.6 \%$ and $12.5 \%$ in comparison with LEACH and GHMAC. For CH nodes, the energy consumption is reduced by $20 \%$ than GHMAC. Thus, these results demonstrate that EEHMAC performs better than LEACH and GHMAC in terms of energy efficiency under various load conditions.

\subsection{Delay Analysis}

In this section, we first study the end-to-end (E2E) delay for both GHMAC and EEHMAC while the traffic load is varied from 0.1 to one erlangs. As clearly seen from Figure 13 and Figure 14, EEHMAC has a smaller delay than GHMAC under all traffic conditions. For increased traffic load, the delay is gradually increased in LEACH and GHMAC. Using CSMA, the probability of getting channel access is reduced in GHMAC due to more number of contenders during maximum traffic load. However in EEHMAC, inter cluster communication is carried out using fixed frequency slots that are assigned during the cluster formation phase. Therefore, the $\mathrm{CH}$ node need not wait for the free channel, and thereby decreasing the delay incurred. For example, EEHMAC reduces E2E delay by $19 \%$ compared with GHMAC when the number of nodes is 900 . Thus, it can be observed that the EEHMAC performance is better than GHMAC in terms of end-to-end delay for all traffic load conditions.

\subsection{Throughput Analysis}

Figure 15 shows the throughput performance comparison of EEHMAC protocol with LEACH and GHMAC

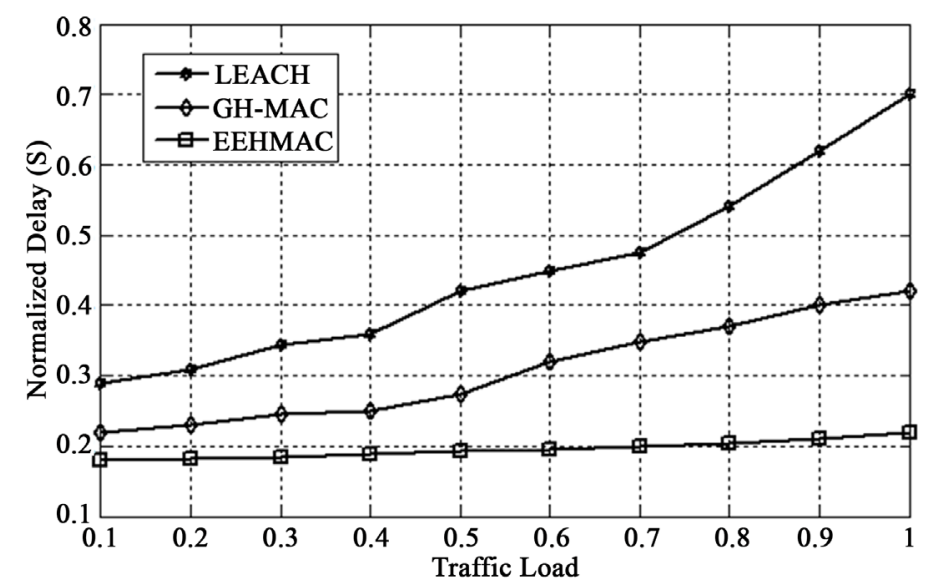

Figure 13. Normalized delay for various traffic load.

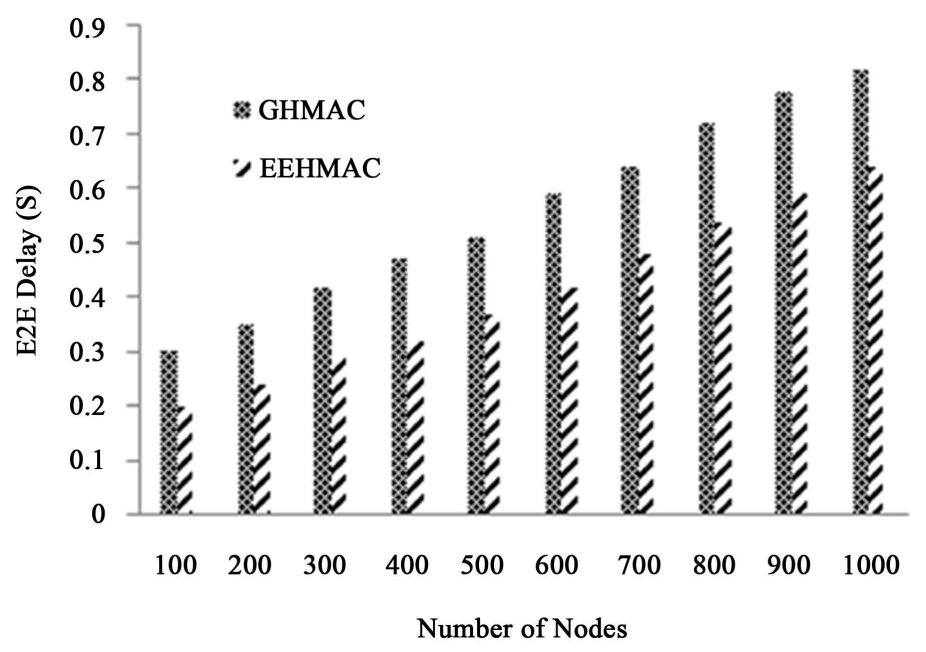

Figure 14. E2E delay Vs density. 


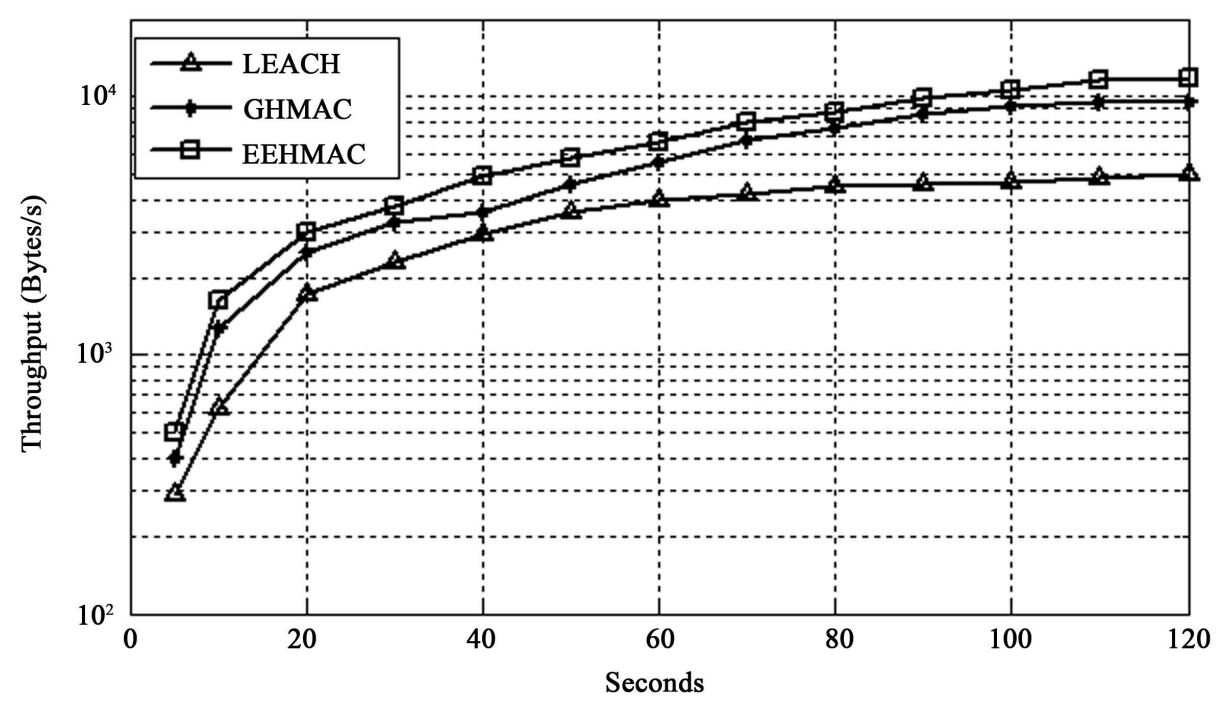

Figure 15. Throughput of different MAC protocols.

protocols for various traffic load when the distance is constant and topology is the same. Compared to LEACH, EEHMAC improved the throughput of network by 56\% and 14\% improvement with GH-MAC. This is due to the collision free characteristics of hybrid EEHMAC protocol. However, the probability of collision is increased in GHMAC if numbers of nodes are increased. This results in reduced throughput of GHMAC. Thus, from the figure it is evident that the best MAC protocol in terms of throughput is EEHMAC than LEACH and GHMAC.

\section{Conclusions}

In this paper, we introduced EEHMAC, an energy efficient hybrid MAC protocol for cluster based wireless sensor networks. EEHMAC allows member nodes of cluster to use TDMA and FDMA for CH nodes. Scheduling mechanism embedded in the MAC protocol helps to achieve this. This scheduling mechanism not only assigns the channel, but also reduces collision problem and thereby achieved the lifetime extension of sensor nodes. The number of frequency slots is limited to number of clusters. By selecting optimum number of clusters for energy efficient operation and by combining TDMA and FDMA techniques for channel accessing, EEHMAC becomes more energy efficient, more robust to topological changes and fairer in resource allocation. It gracefully adapts to network density and improves energy efficiency as well as throughput.

The performances of overall MAC scheme evaluated through simulation have shown a significant improvement in energy efficiency by 56.3\% and $45.23 \%$ compared to LEACH and GHMAC respectively. In comparison with GHMAC, 67.8\% throughput is improved in EEHMAC due to its collision free nature. EEHMAC achieved 19\% reduction in end-to-end delay in comparison with GHMAC.

In future, we aim to achieve further enhancement over EEHMAC through the proposal of a more dynamic and efficient mechanism for transmission power control. We also look forward to implement it over test bed to validate the simulation results provided here.

\section{References}

[1] Mehta, S. and Kwak, K.S. (2007) H-MAC: A Hybrid MAC Protocol for Wireless Sensor Networks. Proceedings of International Technical Conference on Circuits/Systems, Computers and Communications (ITC-CSCC'07), Busan, 8-11 June 2007, 705-706.

[2] Bachir, A., Dohler, M., Watteyne, T. and Leung, K.K. (2010) MAC Essentials for Wireless Sensor Networks. IEEE Communication Surveys and Tutorials, 12, 222-248. http://dx.doi.org/10.1109/SURV.2010.020510.00058

[3] Karl, H. and Willig, A. (2005) Protocols and Architectures for Wireless Sensor Networks. Wiley \& Sons Ltd., Chichester. http://dx.doi.org/10.1002/0470095121

[4] Ye, W., Heidemann, J. and Estrin, D. (2002) An Energy-Efficient MAC Protocol for Wireless Sensor Networks. Proceedings of 21st Annual Joint Conference of the IEEE Computer and Communications Societies, New York, 23-27 June 2002, 1567-1576. http://dx.doi.org/10.1109/INFCOM.2002.1019408 
[5] Van Dam, T. and Langendoen, K. (2003) An Adaptive Energy-Efficient MAC Protocol for Wireless Sensor Networks. Proceedings of 1st International Conference on Embedded Networked Sensor Systems, Los Angeles, 5-7 November 2003, 171-180. http://dx.doi.org/10.1145/958491.958512

[6] Keshavarzian, A., Lee, H. and Venkatraman, L. (2006) Wakeup Scheduling in Wireless Sensor Networks. Proceedings of 7th ACM International Symposium on Mobile Ad-Hoc Networking and computing, Florence, 22-25 May 2006, 322333. http://dx.doi.org/10.1145/1132905.1132941

[7] Jha, M.K., Pandey, A.K., Pal, D. and Mohan, A. (2011) An Energy-Efficient Multi-Layer MAC (ML-MAC) Protocol for Wireless Sensor Networks. AEU-International Journal of Electronics and Communications, 65, 209-216. http://dx.doi.org/10.1016/j.aeue.2010.03.006

[8] Ye, W., Heidemann, J. and Estrin, D. (2004) Medium Access Control with Coordinated Adaptive Sleeping for Wireless Sensor Networks. IEEE/ACM Transactions on Networking, 12, 493-506. http://dx.doi.org/10.1109/TNET.2004.828953

[9] Incel, O.D., Van Hoesel, L., Jansen, P. and Havinga, P. (2011) MC-LMAC: A Multi-Channel MAC Protocol for Wireless Sensor Network. Ad Hoc Networks, 9, 73-94. http://dx.doi.org/10.1016/j.adhoc.2010.05.003

[10] Salajegheh, M., Soroush, H. and Kalis, A. (2007) HYMAC: Hybrid TDMA/FDMA Medium Access Control Protocol for Wireless Sensor Networks. Proceedings of 18th International Symposium on Personal, Indoor and Mobile Radio Communications (PIMRC), Athens, 3-7 September 2007, 1-5. http://dx.doi.org/10.1109/pimrc.2007.4394374

[11] Rhee, I., Warrier, A., Aia, M., Min, J. and Sichitiu, ML. (2008) Z-MAC: A Hybrid MAC for Wireless Sensor Networks. IEEE/ACM Transactions on Networking, 16, 511-524. http://dx.doi.org/10.1109/TNET.2007.900704

[12] Vidhya, J., Kalpana, G, and Dananjayan, P. (2009) Energy Efficient Hybrid MAC Protocol for Cluster-Based Wireless Sensor Network. International Journal of Computer and Electrical Engineering, 1, 1793-8198. http://dx.doi.org/10.7763/ijcee.2009.v1.41

[13] Raja, P. and Dananjayan, P. (2014) Game Theory Based Energy Efficient Hybrid MAC Protocol for Lifetime Enhancement of Wireless Sensor Network. Iranian Journal of Electrical \& Electronic Engineering, 10, 10-17.

[14] Kang, H., Zhao, Y.N. and Mei, F. (2013) A Graph Coloring Based TDMA Scheduling Algorithm for Wireless Sensor Networks. Wireless Personal Communications, 72, 1005-1022. http://dx.doi.org/10.1007/s11277-013-1052-9

[15] Heinzelman, W. (2000) Application-Specific Protocol Architectures for Wireless Networks. Ph.D. Thesis, Massachusetts Institute of Technology, Cambridge, MA.

\section{Submit or recommend next manuscript to SCIRP and we will provide best service for you:}

Accepting pre-submission inquiries through Email, Facebook, LinkedIn, Twitter, etc.

A wide selection of journals (inclusive of 9 subjects, more than 200 journals)

Providing 24-hour high-quality service

User-friendly online submission system

Fair and swift peer-review system

Efficient typesetting and proofreading procedure

Display of the result of downloads and visits, as well as the number of cited articles

Maximum dissemination of your research work

Submit your manuscript at: http://papersubmission.scirp.org/ 